\title{
SUCCESSIVE CARDINALS WITH NO PARTIAL SQUARE
}

\author{
JOHN KRUEGER
}

\begin{abstract}
We construct a model in which for all $1 \leq n<\omega$, there is no stationary subset of $\aleph_{n+1} \cap \operatorname{cof}\left(\aleph_{n}\right)$ which carries a partial square.
\end{abstract}

Let $\kappa$ be an uncountable cardinal and let $\mu \leq \kappa$ be regular. A set $A \subseteq \kappa^{+} \cap \operatorname{cof}(\mu)$ is said to carry a partial square if there exists a sequence $\left\langle c_{\alpha}: \alpha \in A\right\rangle$ such that each $c_{\alpha}$ is club in $\alpha$ with order type $\mu$, and for all $\gamma$ which is a common limit point of $c_{\alpha}$ and $c_{\beta}, c_{\alpha} \cap \gamma=c_{\beta} \cap \gamma$. Such a sequence is called a partial square sequence.

It was shown by Shelah [6] that if $\mu<\kappa$ are regular cardinals, then there exists a stationary subset of $\kappa^{+} \cap \operatorname{cof}(\mu)$ which carries a partial square. In a model of Magidor [4] which satisfies a strong form of stationary set reflection, there is no stationary subset of $\aleph_{2} \cap \operatorname{cof}\left(\aleph_{1}\right)$ which carries a partial square (this was pointed out by several authors; see [7] and [5]). The exact consistency result was obtained in [3], where we showed that the existence of a greatly Mahlo cardinal is equiconsistent with the statement that for some regular uncountable cardinal $\kappa$, there is no stationary subset of $\kappa^{+} \cap \operatorname{cof}(\kappa)$ which carries a partial square.

In this paper we demonstrate how to obtain models in which there are successive cardinals with no partial square. Specifically, starting with an increasing sequence $\left\langle\kappa_{n}: 1 \leq n<\omega\right\rangle$ of supercompact cardinals, we collapse each $\kappa_{n}$ to become $\aleph_{n+1}$ in such a way that in the final model, for all $1 \leq n<\omega$ there is no stationary subset of $\aleph_{n+1} \cap \operatorname{cof}\left(\aleph_{n}\right)$ which carries a partial square.

\section{The BAsic IDEA}

Let $\kappa$ be a regular uncountable cardinal. To obtain a model with no stationary subset of $\kappa^{+} \cap \operatorname{cof}(\kappa)$ carrying a partial square, we first Lévy collapse a greatly Mahlo cardinal $\lambda>\kappa$ to become $\kappa^{+}$, and then iterate to kill the stationarity of all subsets of $\kappa^{+}$which carry a partial square (see [3]). The purpose of the Lévy collapse is to prepare the ground model so that the iteration of club adding preserves $\kappa^{+}$.

Suppose we would like to continue further and force that there is no stationary subset of $\kappa^{++} \cap \operatorname{cof}\left(\kappa^{+}\right)$which carries a partial square. A naive approach would be to Lévy collapse another large cardinal $\mu>\lambda=\kappa^{+}$to become $\kappa^{++}$, and then iterate to kill subsets of $\kappa^{++} \cap \operatorname{cof}\left(\kappa^{+}\right)$which carry a partial square. The problem is that the Lévy collapse of $\mu$ to become $\kappa^{++}$adds subsets of $\kappa^{+}$, and hence could add a stationary subset of $\kappa^{+} \cap \operatorname{cof}(\kappa)$ which carries a partial square, thereby ruining what was achieved in the first construction (see Section 5).

A solution is that instead of first forcing no partial square on $\kappa^{+}$and then collapsing $\mu$ to become $\kappa^{++}$, we blend the two forcings together. In other words, we iterate with $<\kappa^{+}$-support alternating between collapsing cardinals above $\kappa^{+}$and

2010 Mathematical Subject Classification: 03E55, 03E35.

Key words and phrases: partial square sequence. 
adding club subsets of $\kappa^{+}$. It turns out that the Lévy collapse which made $\lambda$ into $\kappa^{+}$prepares the ground model so that this iteration preserves $\kappa^{+}$. Moreover, this iteration itself serves as a preparation for the next iteration of adding club subsets of $\kappa^{++}$. This process can be continued $\omega$ many steps to obtain the consistency result which was described in the introductory section of the paper.

\section{The Forcing Iteration}

Fix a regular uncountable cardinal $\kappa$ and a supercompact cardinal $\lambda>\kappa$. We define a $<\kappa^{+}$-support forcing iteration of length $\lambda$. The purpose of this iteration is threefold. First, we would like to add club subsets of $\kappa^{+}$so that in the final model any subset of $\kappa^{+} \cap \operatorname{cof}(\kappa)$ which carries a partial square is non-stationary. Second, we would like to collapse $\lambda$ to become $\kappa^{++}$. Third, we would like to prepare the ground model so that we can destroy stationary subsets of $\kappa^{++} \cap \operatorname{cof}\left(\kappa^{+}\right)$which carry a partial square without collapsing $\kappa^{++}$.

The main challenge is to ensure that $\kappa^{+}$is not collapsed by this forcing iteration. This is achieved by preparing the ground model with either a Lévy collapse or a forcing iteration of the same form as we are describing now but on smaller cardinals. The preservation of $\kappa^{+}$is demonstrated in the next section. For now we just assume $\kappa^{+}$is preserved by the forcings we describe in order for the definition of the forcing iteration to make sense.

Fix a Laver function $l: \lambda \rightarrow V_{\lambda}$. We define a forcing iteration

$$
\left\langle\mathbb{P}_{i}, \dot{\mathbb{Q}}_{j}: i \leq \lambda, j<\lambda\right\rangle
$$

by induction.

Let $\mathbb{P}_{0}$ be the trivial forcing. Now assume that a limit ordinal $\delta \leq \lambda$ is given and $\mathbb{P}_{i}$ is defined for all $i<\delta$. Define $\mathbb{P}_{\delta}$ as the forcing poset consisting of all functions $p$ with $\operatorname{dom}(p) \subseteq \delta$ and $|\operatorname{dom}(p)|<\kappa^{+}$such that for all $i<\delta, p\left\lceil i \in \mathbb{P}_{i}\right.$. The ordering on $\mathbb{P}_{\delta}$ is defined by letting $q \leq p$ if for all $i<\delta, q \uparrow i \leq p \uparrow i$ in $\mathbb{P}_{i}$. (Equivalently, $q \leq p$ if $\operatorname{dom}(p) \subseteq \operatorname{dom}(q)$ and for all $i \in \operatorname{dom}(p), q \uparrow i \Vdash q(i) \leq p(i)$ in $\dot{\mathbb{Q}}_{i}$.)

Now let $\alpha<\lambda$ and assume that $\mathbb{P}_{\alpha}$ is defined. The definition of $\dot{\mathbb{Q}}_{\alpha}$ will depend on which of the following cases hold.

Case 1: $\alpha$ is an inaccessible cardinal larger than $\kappa^{+}$, and for all $\beta<\alpha,\left|\mathbb{P}_{\beta}\right|<\alpha$.

\section{Case 2: Not Case 1.}

In Case 2 , let $\dot{\mathbb{Q}}_{\alpha}$ be a $\mathbb{P}_{\alpha}$-name for the collapse $\operatorname{CoLL}\left(\kappa^{+}, \kappa^{++}\right)$.

Assume Case 1. Then by standard arguments, $\mathbb{P}_{\alpha}$ is $\alpha$-c.c. Since Case 2 occurs cofinally below $\alpha$, all cardinals in the open interval $\left(\kappa^{+}, \alpha\right)$ are collapsed by $\mathbb{P}_{\alpha}$. Hence if $\mathbb{P}_{\alpha}$ preserves $\kappa^{+}$, an assumption which will be justified in the next section, then $\mathbb{P}_{\alpha}$ collapses $\alpha$ to become $\kappa^{++}$.

We consider three subcases.

Subcase 1a: $l(\alpha)=\theta$ for some regular cardinal $\theta$ larger than $\alpha$.

In this case, let $\dot{\mathbb{Q}}_{\alpha}$ be a $\mathbb{P}_{\alpha}$-name for the collapse $\operatorname{CoLL}\left(\kappa^{+}, \theta\right)$.

Subcase 1b: $l(\alpha)=\dot{S}$ for some $\mathbb{P}_{\alpha}$-name $\dot{S}$ such that $\mathbb{P}_{\alpha}$ forces that $\dot{S}$ is a subset of $\kappa^{+} \cap \operatorname{cof}(\kappa)$ which carries a partial square. 
In this case, let $\dot{\mathbb{Q}}_{\alpha}$ be a $\mathbb{P}_{\alpha}$-name for $\mathbb{P}(\dot{S})$, where $\mathbb{P}(\dot{S})$ is the forcing poset for adding a club subset of $\kappa^{+}$which is disjoint from $\dot{S}$, ordered by end-extension. Specifically, $\mathbb{P}_{\alpha}$ forces that a set $c$ is in $\mathbb{P}(\dot{S})$ if $c$ is a closed, bounded subset of $\kappa^{+}$ disjoint from $\dot{S}$, and $d \leq c$ in $\mathbb{P}(\dot{S})$ if $d \cap(\max (c)+1)=c$. It is not hard to see that $\mathbb{P}(\dot{S})$ is forced to be $\kappa$-closed.

Subcase 1c: Neither Subcase 1a nor Subcase 1b.

In this case, we let $\dot{\mathbb{Q}}_{\alpha}$ be a $\mathbb{P}_{\alpha}$-name for the collapse $\operatorname{CoLL}\left(\kappa^{+}, \kappa^{++}\right)$.

Note that in all cases, $\mathbb{P}_{\alpha}$ forces that $\dot{\mathbb{Q}}_{\alpha}$ is $\kappa$-closed. Therefore the forcing iteration is $\kappa$-closed. Thus all cardinals and cofinalities less than or equal to $\kappa$ are preserved.

Let us assume that the iteration $\mathbb{P}_{\lambda}$ preserves $\kappa^{+}$, and we show it forces that there is no stationary subset of $\kappa^{+} \cap \operatorname{cof}(\kappa)$ which carries a partial square. Since $\lambda$ is inaccessible, $\mathbb{P}_{\lambda}$ is $\lambda$-c.c., and $\mathbb{P}_{\lambda}$ collapses $\lambda$ to become $\kappa^{++}$.

Consider a $\mathbb{P}_{\lambda}$-name for a subset of $\kappa^{+} \cap \operatorname{cof}(\kappa)$ which carries a partial square. Since a partial square sequence can easily be coded as a subset of $\kappa^{+}$, we can choose a nice $\mathbb{P}_{\lambda}$-name $\dot{S}$ for the set and also a nice $\mathbb{P}_{\lambda}$-name for the partial square sequence on $\dot{S}$. As $\mathbb{P}_{\lambda}$ is $\lambda$-c.c., there is $\gamma<\lambda$ such that these names are $\mathbb{P}_{\gamma}$-names. Now being a partial square sequence is upwards absolute, so for all $\gamma \leq \beta \leq \lambda, \dot{S}$ is a $\mathbb{P}_{\beta}$-name and $\mathbb{P}_{\beta}$ forces that $\dot{S}$ carries a partial square.

Since $l$ is a Laver function and $\dot{S} \in V_{\lambda}$, an easy argument shows that there are stationarily many inaccessibles $\alpha<\lambda$ such that $l(\alpha)=\dot{S}$. As Case 1 holds for club many inaccessibles $\alpha<\lambda$, we can find $\alpha \geq \gamma$ such that Case 1 holds for $\alpha$ and $l(\alpha)=\dot{S}$. Then Subcase $1 \mathrm{~b}$ holds at $\alpha$. So $\dot{\mathbb{Q}}_{\alpha}=\mathbb{P}(\dot{S})$. Therefore $\mathbb{P}_{\alpha+1}$ forces that there is a club subset of $\kappa^{+}$which is disjoint from $\dot{S}$. Hence $\mathbb{P}_{\lambda}$ forces that $\dot{S}$ is non-stationary.

\section{Preserving Cardinals}

Assume that we are given regular cardinals

$$
\kappa<\kappa^{+}<\lambda<\mu,
$$

where $\lambda$ and $\mu$ are supercompact.

We consider a two-step iteration $\mathbb{P}_{\lambda} * \mathbb{P}_{\mu}$. After forcing with $\mathbb{P}_{\lambda}, \lambda$ will be collapsed to become $\kappa^{++}$. Then $\mathbb{P}_{\mu}$ is the $<\kappa^{++}$-support forcing iteration of length $\mu$ which collapses $\mu$ to become $\kappa^{+++}$, and forces that there is no stationary subset of $\kappa^{++} \cap \operatorname{cof}\left(\kappa^{+}\right)$which carries a partial square. The reason for forcing with $\mathbb{P}_{\lambda}$ is to prepare the ground model so that $\mathbb{P}_{\mu}$ preserves $\kappa^{++}$.

The forcing poset $\mathbb{P}_{\lambda}$ can take two forms. First, $\mathbb{P}_{\lambda}$ can be the Lévy collapse $\operatorname{ColL}\left(\kappa^{+},<\lambda\right)$. Second, $\mathbb{P}_{\lambda}$ can be the $<\kappa^{+}$-support forcing iteration which collapses $\lambda$ to become $\kappa^{++}$while forcing that there is no stationary subset of $\kappa^{+} \cap \operatorname{cof}(\kappa)$ which carries a partial square. In the second case, we make two additional assumptions: that $\kappa$ is uncountable, and that $\mathbb{P}_{\lambda}$ preserves $\kappa^{+}$.

We will prove that $\mathbb{P}_{\mu}$ preserves $\kappa^{++}$after forcing with $\mathbb{P}_{\lambda}$. Since the first case that $\mathbb{P}_{\lambda}$ is the Lévy collapse is similar to, but somewhat easier than, the second case, we will provide the complete details only for the second case. We include some comments as to how the proof can be modified to handle the Lévy collapse.

We will use the following two results. 
Theorem 3.1 (Magidor [4]). Let $\nu$ be a regular uncountable cardinal, and let $\mathbb{P}$ be a $\nu$-closed forcing poset. Suppose $\theta \geq|\mathcal{P}(\mathbb{P})|$. Then $\operatorname{ColL}(\nu, \theta)$ factors as $\mathbb{P} * \mathbb{Q}$, where $\mathbb{P}$ forces that $\mathbb{Q}$ is $\nu$-closed.

Proposition $3.2([3])$. Let $\mu$ be an ordinal of uncountable cofinality, and let $\left\langle d_{\gamma}\right.$ : $\gamma<\mu\rangle$ be a sequence such that $d_{\gamma} \subseteq \gamma$ for all $\gamma<\mu$. Let $\mathbb{P}$ be a proper forcing poset. Then $\mathbb{P}$ forces that if $c$ is a club subset of $\mu$ such that for all $\gamma \in \lim (c)$, $c \cap \gamma=d_{\gamma}$, then $c$ is in the ground model.

Fix a Laver function $l_{0}: \lambda \rightarrow V_{\lambda}$. Let

$$
\left\langle\mathbb{P}_{i}^{0}, \dot{\mathbb{Q}}_{j}^{0}: i \leq \lambda, j<\lambda\right\rangle
$$

be the $<\kappa^{+}$-support forcing iteration defined in the previous section using $l_{0}$ which collapses $\lambda$ to become $\kappa^{++}$and forces that there is no stationary subset of $\kappa^{+} \cap \operatorname{cof}(\kappa)$ which carries a partial square. We assume that this iteration preserves $\kappa^{+}$. Let $\mathbb{P}_{\lambda}=\mathbb{P}_{\lambda}^{0}$.

Let $G_{\lambda}$ be a generic filter on $\mathbb{P}_{\lambda}$ over $V$. Then in $V\left[G_{\lambda}\right], \lambda=\kappa^{++}$and $\mu$ is supercompact. Working in this model, fix a Laver function $l_{1}: \mu \rightarrow V_{\mu}$, and let

$$
\left\langle\mathbb{P}_{i}^{1}, \dot{\mathbb{Q}}_{j}^{1}: i \leq \mu, j<\mu\right\rangle
$$

be the $<\kappa^{++}$-support forcing iteration defined in the previous section using $l_{1}$. This forcing iteration is $\kappa^{+}$-closed, and assuming that it preserves $\kappa^{++}$, which we will prove below, it collapses $\mu$ to become $\kappa^{+++}$and forces that there is no stationary subset of $\kappa^{++} \cap \operatorname{cof}\left(\kappa^{+}\right)$which carries a partial square.

To show that the iteration $\mathbb{P}_{\mu}^{1}$ preserves $\kappa^{++}$, we will prove by induction on $\alpha \leq \mu$ that $\mathbb{P}_{\alpha}^{1}$ is $\kappa^{++}$-distributive. So fix $\alpha \leq \mu$, and assume as an induction hypothesis that for all $\beta<\alpha, \mathbb{P}_{\beta}^{1}$ is $\kappa^{++}$-distributive. Let $\mathcal{D}$ be a family of fewer than $\kappa^{++}$ many dense open subsets of $\mathbb{P}_{\alpha}^{1}$, and let $p \in \mathbb{P}_{\alpha}^{1}$. We will prove that there exists a condition $q \leq p$ in the intersection $\bigcap \mathcal{D}$.

Fix a regular cardinal $\theta$ large enough so that $\lambda, \mathbb{P}_{\alpha}^{1}$, and $\mathcal{P}\left(\mathbb{P}_{\alpha}^{1}\right)$ are in $H(\theta)$. Since $l_{0}$ is a Laver function for $\lambda$, we can fix an elementary embedding $j: V \rightarrow M$ with critical point $\lambda$ such that $M^{\theta} \subseteq M, j(\lambda)>\theta$, and $j\left(l_{0}\right)(\lambda)=\theta$.

Consider in $M$ stage $\lambda$ of the forcing iteration $j\left(\left\langle\mathbb{P}_{i}^{0}, \dot{\mathbb{Q}}_{j}^{0}: i \leq \lambda, j<\lambda\right\rangle\right)$. Since $j\left(\mathbb{P}_{\lambda}^{0}\right) \uparrow \lambda=\mathbb{P}_{\lambda}^{0}$, clearly Case 1 is satisfied in the definition of the forcing iteration given in the previous section. As $j\left(l_{0}\right)(\lambda)=\theta$, and $\theta$ is a regular cardinal larger than $\lambda$ in $M$, Subcase 1a is satisfied.

So by the closure of $M$ we can factor $j\left(\mathbb{P}_{\lambda}^{0}\right)$ as

$$
j\left(\mathbb{P}_{\lambda}^{0}\right)=\mathbb{P}_{\lambda}^{0} * \operatorname{CoLL}\left(\kappa^{+}, \theta\right) * \mathbb{P}_{\lambda+1, j(\lambda)}^{0} .
$$

Since $\mathbb{P}_{\alpha}^{1}$ is $\kappa^{+}$-closed in $M\left[G_{\lambda}\right]$ and $\theta>\left|\mathcal{P}\left(\mathbb{P}_{\alpha}^{1}\right)\right|$, we can apply Magidor's Theorem 3.1 to get

$$
j\left(\mathbb{P}_{\lambda}^{0}\right)=\mathbb{P}_{\lambda}^{0} * \mathbb{P}_{\alpha}^{1} * \mathbb{Q} * \mathbb{P}_{\lambda+1, j(\lambda)}^{0},
$$

where $\mathbb{Q}$ is forced to be $\kappa^{+}$-closed. Then $\mathbb{P}_{\lambda}^{0} * \mathbb{P}_{\alpha}^{1}$ forces that $\mathbb{Q} * \mathbb{P}_{\lambda+1, j(\lambda)}^{0}$ is $\kappa$-closed.

Remark: In the case that $\mathbb{P}_{\lambda}$ is the Lévy collapse, we use a similar factorization. In that case, choose $\theta$ as above, and let $j: V \rightarrow M$ be an elementary embedding with critical point $\lambda$ such that $M^{\theta} \subseteq M$ and $j(\lambda)>\theta$. Then $j\left(\mathbb{P}_{\lambda}\right)=\operatorname{CoLL}\left(\kappa^{+}, j(\lambda)\right)$. So using Magidor's Theorem we can factor $j\left(\mathbb{P}_{\lambda}\right)$ as $\mathbb{P}_{\lambda} * \mathbb{P}_{\alpha}^{1} * \mathbb{Q} * \mathbb{R}$, where $\mathbb{Q} * \mathbb{R}$ is forced to be $\kappa^{+}$-closed. In either case, the quotient $j\left(\mathbb{P}_{\lambda}\right) / \mathbb{P}_{\lambda} * \mathbb{P}_{\alpha}^{1}$ is $\aleph_{1}$-closed, and hence proper. 
Let us generically extend the elementary embedding $j: V \rightarrow M$ to $V\left[G_{\lambda}\right]$. So choose a generic filter $H_{\alpha} * I * J$ for $\mathbb{P}_{\alpha}^{1} * \mathbb{Q} * \mathbb{P}_{\lambda+1, j(\lambda)}^{1}$ over $V\left[G_{\lambda}\right]$ such that $p \in H_{\alpha}$. Since $j\left[G_{\lambda}\right]=G_{\lambda} \subseteq G_{\lambda} * H_{\alpha} * I * J$, in the model $V\left[G_{\lambda} * H_{\alpha} * I * J\right]$ we can extend $j$ to $j: V\left[G_{\lambda}\right] \rightarrow M\left[G_{\lambda} * H_{\alpha} * I * J\right]$ such that $j\left(G_{\lambda}\right)=G_{\lambda} * H_{\alpha} * I * J$. (In the case that $\mathbb{P}_{\lambda}$ is the Lévy collapse, we extend $j$ in a similar manner).

So we have a generic elementary embedding $j: V\left[G_{\lambda}\right] \rightarrow M\left[j\left(G_{\lambda}\right)\right]$. By the elementarity of $j$, to prove that there exists a condition below $p$ in $\bigcap \mathcal{D}$, it suffices to show in $M\left[j\left(G_{\lambda}\right)\right]$ that there is a condition $q \leq j(p)$ in $\bigcap j(\mathcal{D})$. Since $|\mathcal{D}|<\lambda$, $j(\mathcal{D})=j[\mathcal{D}]$. So it suffices to construct in $M\left[j\left(G_{\lambda}\right)\right]$ a condition $q \leq j(p)$ which is in $j(D)$ for all $D \in \mathcal{D}$.

By the closure of $M$, standard arguments show that $j\left\lceil H(\theta)^{V\left[G_{\lambda}\right]}\right.$ is in $M\left[G_{\lambda}\right]$. Thus the set $j\left[H_{\alpha}\right]$ is a member of $M\left[j\left(G_{\lambda}\right)\right]$. We will use the set $j\left[H_{\alpha}\right]$ to construct the condition $q$.

By standard facts about iterated forcing, a set $s$ is in $\mathbb{P}_{\alpha}^{1}$ provided that $s$ is a function whose domain is a subset of $\alpha$ of size less than $\lambda$, and for all $\gamma$ in $\operatorname{dom}(s)$, if $s\left\lceil\gamma \in \mathbb{P}_{\gamma}^{1}\right.$ then $s \Vdash s(\gamma) \in \dot{\mathbb{Q}}_{\gamma}^{1}$. For then it can be shown that $s \uparrow \beta \in \mathbb{P}_{\beta}^{1}$ by induction on $\beta \leq \alpha$.

We define $q$ in $j\left(\mathbb{P}_{\alpha}^{1}\right)$ with domain $j[\alpha]$. In $M\left[j\left(G_{\lambda}\right)\right],|j[\alpha]|=|\alpha| \leq \kappa^{+}<j(\lambda)$, so the domain of $q$ is of the correct form. By the preceding comments, it suffices to define $q(j(\beta))$ by induction on $\beta<\alpha$ so that

$$
q\left\lceilj ( \beta ) \in j ( \mathbb { P } _ { \beta } ^ { 1 } ) \Longrightarrow q \left\lceil j(\beta) \Vdash q(j(\beta)) \in j\left(\dot{\mathbb{Q}}_{\beta}^{1}\right) .\right.\right.
$$

We would also like $q$ to be below $j\left[H_{\alpha}\right]$. So we will maintain by induction on $\beta \leq \alpha$ that $q\left\lceil j(\beta)\right.$ is below $j\left[H_{\beta}\right]$, where $H_{\beta}=\left\{s\left\lceil\beta: s \in H_{\alpha}\right\}\right.$. Note that $q\lceil j(\beta)$ is below $j\left[H_{\beta}\right]$ provided that for all $\gamma \leq \beta$, if $q\left\lceil j(\gamma)\right.$ is below $j\left[H_{\gamma}\right]$ then for all $s \in H_{\alpha}, q\lceil j(\gamma) \Vdash q(j(\gamma)) \leq j(s(\gamma))$.

So let $\beta<\alpha$ be given, and assume that $q\left\lceil j(\beta)\right.$ is in $j\left(\mathbb{P}_{\beta}^{1}\right)$ and is below $j\left[H_{\beta}\right]$. We claim that there is a $j\left(\mathbb{P}_{\beta}^{1}\right)$-name $\dot{a}$ for a condition in $j\left(\dot{\mathbb{Q}}_{\beta}^{1}\right)$ such that $q\lceil j(\beta)$ forces $\dot{a} \leq j(s(\beta))$ for all $s \in H_{\alpha}$. Then we let $q(j(\beta))=\dot{a}$ and we are finished. Suppose for a contradiction this is false. Then there is a condition $r \leq q\lceil j(\beta)$ in $j\left(\mathbb{P}_{\beta}^{1}\right)$ which forces over $M\left[j\left(G_{\lambda}\right)\right]$ that the set $\left\{j(s(\beta)): s \in H_{\alpha}\right\}$ has no lower bound in $j\left(\dot{\mathbb{Q}}_{\beta}^{1}\right)$. Fix a generic filter $K_{\beta}$ for $j\left(\mathbb{P}_{\beta}^{1}\right)$ over $V\left[G_{\lambda} * H_{\alpha} * I * J\right]$ which contains $r$.

Now $H_{\beta}$ is a generic filter for $\mathbb{P}_{\beta}^{1}$ over $V\left[G_{\lambda}\right]$. Since $r \leq q, q$ is below $j\left[H_{\beta}\right]$, and $r$ is in $K_{\beta}$, it follows that in $V\left[G_{\lambda} * H_{\alpha} * I * J * K_{\beta}\right]$ we can lift $j$ to $j: V\left[G_{\lambda} * H_{\beta}\right] \rightarrow$ $M\left[G_{\lambda} * H_{\alpha} * I * J * K_{\beta}\right]$ such that $j\left(G_{\lambda} * H_{\beta}\right)=G_{\lambda} * H_{\alpha} * I * J * K_{\beta}$.

We will abuse notation and write $s(\beta)$ for $s(\beta)^{H_{\beta}}$ for $s \in H_{\alpha}$. Let $H(\beta)=$ $\left\{s(\beta): s \in H_{\alpha}\right\}$, which is a generic filter for $\mathbb{Q}_{\beta}^{1}$ over $V\left[G_{\lambda} * H_{\beta}\right]$. Then $j[H(\beta)]=$ $\left\{j(s(\beta)): s \in H_{\alpha}\right\}$ has no lower bound in $j\left(\mathbb{Q}_{\beta}^{1}\right)$. Note that the set $j[H(\beta)]$ is directed and has size $\kappa^{+}$in $M\left[j\left(G_{\lambda} * H_{\beta}\right)\right]$.

We consider the two possibilities for the forcing poset $\mathbb{Q}_{\beta}^{1}$. On the one hand, $\mathbb{Q}_{\beta}^{1}$ can be a Lévy collapse of the form $\operatorname{ColL}(\lambda, \xi)$ for some $\xi$, as in Case 2 and Subcases $1 \mathrm{a}$ and $1 \mathrm{c}$ in the definition of the forcing iteration. In that case, $j\left(\mathbb{Q}_{\beta}^{1}\right)$ is $j(\lambda)$-directed closed. But $j[H(\beta)]$ is directed and has size less than $j(\lambda)$, and therefore has a lower bound, which is a contradiction.

Let us assume the other case that Subcase $1 \mathrm{~b}$ holds for $\beta$. Then in $V\left[G_{\lambda}\right], \beta$ is strongly inaccessible and for all $\gamma<\beta,\left|\mathbb{P}_{\gamma}^{1}\right|<\beta$. So $\mathbb{P}_{\beta}^{1}$ is $\beta$-c.c. By the induction 
hypothesis, $\mathbb{P}_{\beta}^{1}$ is $\kappa^{++}$-distributive. Therefore $\mathbb{P}_{\beta}^{1}$ collapses $\beta$ to become $\kappa^{+++}$. By the definition of $\mathbb{Q}_{\beta}^{1}$ in Subcase $1 \mathrm{~b}, \mathbb{Q}_{\beta}^{1}$ is equal to $\mathbb{P}(S)$, where $S$ is a subset of $\kappa^{++} \cap \operatorname{cof}\left(\kappa^{+}\right)$which carries a partial square.

Fix a partial square sequence $\left\langle c_{\nu}: \nu \in S\right\rangle$ in $V\left[G_{\lambda} * H_{\beta}\right]$. Write $j\left(\left\langle c_{\nu}: \nu \in S\right\rangle\right)=$ $\left\langle c_{\nu}^{j}: \nu \in j(S)\right\rangle$. Note that $j(S) \cap \lambda=S$ and $c_{\nu}^{j}=j\left(c_{\nu}\right)=c_{\nu}$ for all $\nu \in S$. So $\left\langle c_{\nu}^{j}: \nu \in j(S)\right\rangle \uparrow \lambda=\left\langle c_{\nu}: \nu \in S\right\rangle$.

Recall that $H(\beta)=\left\{s(\beta): s \in H_{\alpha}\right\}$ is a generic filter for $\mathbb{P}(S)$ over $V\left[G_{\lambda} * H_{\beta}\right]$, and we are assuming that in $M\left[j\left(G_{\lambda} * H_{\beta}\right)\right], j[H(\beta)]$ has no lower bound. Since the critical point of $j$ is $\lambda$, and each condition in $H(\beta)$ is a bounded subset of $\lambda$, $j[H(\beta)]=H(\beta)$. By the genericity of $H(\beta)$, it follows that the set

$$
d=\bigcup j[H(\beta)] \cup\{\lambda\}
$$

is a closed, bounded subset of $j(\lambda)$ with maximum element $\lambda$ such that $d \cap \lambda$ is disjoint from $j(S)$. Also $d$ end-extends $e$ for all $e \in j[H(\beta)]$. Since the set $j[H(\beta)]$ has no lower bound in $j(\mathbb{P}(S)), d$ cannot be a condition, and therefore $\lambda$ must be in $j(S)$.

Consider the set $c_{\lambda}^{j}$, which is a club subset of $\lambda$ in $M\left[j\left(G_{\lambda} * H_{\beta}\right)\right]$ of order type $\kappa^{+}$. For all $\gamma \in \lim \left(c_{\lambda}^{j}\right), c_{\lambda}^{j} \cap \gamma=c_{\gamma}^{j}=c_{\gamma}$. But

$$
M\left[j\left(G_{\lambda} * H_{\beta}\right)\right]=M\left[G_{\lambda} * H_{\beta} *\left(H_{\alpha} / H_{\beta}\right) * I * J * K_{\beta}\right],
$$

which is a generic extension of $M\left[G_{\lambda} * H_{\beta}\right]$ by the forcing poset

$$
\mathbb{P}_{\alpha}^{1} / \mathbb{P}_{\beta}^{1} * \mathbb{Q} * \mathbb{P}_{\lambda+1, j(\lambda)}^{0} * j\left(\mathbb{P}_{\beta}^{1}\right),
$$

which is $\kappa$-closed. Since $\kappa$ is uncountable, this forcing poset is proper. Thus by Proposition 3.2, $c_{\lambda}^{j}$ is in $M\left[G_{\lambda} * H_{\beta}\right]$. But $c_{\lambda}^{j}$ is a club subset of $\lambda$ with order type $\kappa^{+}$. This is a contradiction as $\lambda=\kappa^{++}$in $M\left[G_{\lambda} * H_{\beta}\right]$. Remark: A similar argument is made in the case that $\mathbb{P}_{\lambda}$ is the Lévy collapse, using the factorization of $j\left(\mathbb{P}_{\lambda}\right)$ described above.

This completes the construction of $q$. Since $q$ is below $j\left[H_{\alpha}\right]$ and $p \in H_{\alpha}$, $q \leq j(p)$. Also for each $D \in \mathcal{D}$, by genericity we can fix $s \in D \cap H_{\alpha}$. Then $j(s) \in j(D) \cap j\left[H_{\alpha}\right]$. As $q \leq j(s)$ and $j(D)$ is open, $q \in j(D)$. Thus $q \in \bigcap j[\mathcal{D}]$, which completes the proof.

\section{The Consistency Result}

Fix an increasing sequence of supercompact cardinals $\left\langle\kappa_{n}: 1 \leq n<\omega\right\rangle$. Let $\kappa_{0}=\aleph_{1}$. We will define a forcing poset $\mathbb{P}_{\omega}$ which forces that for all $n<\omega$, $\kappa_{n}=\aleph_{n+1}$, and for all $1 \leq n<\omega$ there is no stationary subset of $\aleph_{n+1} \cap \operatorname{cof}\left(\aleph_{n}\right)$ which carries a partial square. We will define $\mathbb{Q}_{n}$ by induction on $n<\omega$, and let $\mathbb{P}_{\omega}$ be the full-support limit of the finite-step iterations $\mathbb{Q}_{0} * \cdots * \mathbb{Q}_{n}$.

Let $\mathbb{Q}_{0}$ be the Lévy collapse $\operatorname{CoLL}\left(\kappa_{0},<\kappa_{1}\right)$. So $\mathbb{Q}_{0}$ collapses $\kappa_{1}$ to become $\aleph_{2}$. Let $\mathbb{Q}_{1}$ be a $\mathbb{Q}_{0}$-name for the $<\kappa_{1}$-support forcing iteration from Section 2 which collapses $\kappa_{2}$ to become $\kappa_{1}^{+}=\aleph_{3}$ and forces that there is no stationary subset of $\kappa_{1} \cap \operatorname{cof}\left(\kappa_{0}\right)=\aleph_{2} \cap \operatorname{cof}\left(\aleph_{1}\right)$ which carries a partial square. Since $\mathbb{Q}_{1}$ was preceded by the Lévy collapse of a supercompact cardinal, $\mathbb{Q}_{1}$ is forced to be $\kappa_{1}$-distributive by the material from the previous section.

Let $n>0$ and suppose we have defined $\mathbb{Q}_{0}, \ldots, \mathbb{Q}_{n}$. Assume as an induction hypothesis that the finite-step iteration $\mathbb{Q}_{0} * \cdots * \mathbb{Q}_{n-1}$ forces that $(1) \kappa_{m}=\aleph_{m+1}$ for all $m \leq n,(2) \mathbb{Q}_{n}$ is $\kappa_{n}$-distributive, and (3) $\mathbb{Q}_{n}$ is the $<\kappa_{n}$-support forcing 
iteration which collapses $\kappa_{n+1}$ to become $\kappa_{n}^{+}=\aleph_{n+2}$ and forces that there is no stationary subset of $\kappa_{n} \cap \operatorname{cof}\left(\kappa_{n-1}\right)=\aleph_{n+1} \cap \operatorname{cof}\left(\aleph_{n}\right)$ which carries a partial square.

Let $\mathbb{Q}_{n+1}$ be a $\mathbb{Q}_{0} * \cdots * \mathbb{Q}_{n}$-name for the $<\kappa_{n+1}$-support forcing iteration which collapses $\kappa_{n+2}$ to become $\kappa_{n+1}^{+}=\aleph_{n+3}$ and forces that there is no stationary subset of $\kappa_{n+1} \cap \operatorname{cof}\left(\kappa_{n}\right)=\aleph_{n+2} \cap \operatorname{cof}\left(\aleph_{n+1}\right)$ which carries a partial square. By the material from the previous section, $\mathbb{Q}_{n+1}$ is forced to be $\kappa_{n+1}$-distributive.

This completes the definition. The induction hypothesis implies that for all $n<\omega, \mathbb{Q}_{0} * \cdots * \mathbb{Q}_{n}$ forces that (a) $\kappa_{m}=\aleph_{m+1}$ for all $m \leq n+1$, (b) $\mathbb{Q}_{n+1}$ is $\kappa_{n}$-closed and $\kappa_{n+1}$-distributive, and collapses $\kappa_{n+2}$ to become $\aleph_{n+3}$, and (c) $\mathbb{Q}_{n+1}$ forces that there is no stationary subset of $\aleph_{n+2} \cap \operatorname{cof}\left(\aleph_{n+1}\right)$ which carries a partial square.

Define $\mathbb{P}_{\omega}$ as the full-support iteration of these finite-step iterations. Specifically, a condition in $\mathbb{P}_{\omega}$ is a function $p$ with domain $\omega$ such that for all $1 \leq n<\omega$, $p\left\lceil n \in \mathbb{Q}_{0} * \cdots * \mathbb{Q}_{n-1}\right.$. Let $q \leq p$ in $\mathbb{P}_{\omega}$ if $q(0) \leq p(0)$ in $\mathbb{Q}_{0}$ and for all $1 \leq n<\omega$, $q \uparrow n$ forces that $q(n) \leq p(n)$ in $\mathbb{Q}_{n}$.

Standard arguments show that for all $n<\omega$, we can factor $\mathbb{P}_{\omega}$ as

$$
\mathbb{P}_{\omega}=\mathbb{Q}_{0} * \cdots * \mathbb{Q}_{n} * \mathbb{P}_{n+1, \omega},
$$

where $\mathbb{P}_{n+1, \omega}$ is forced to be $\kappa_{n}$-closed.

Let us prove that $\mathbb{P}_{\omega}$ forces that for all $n<\omega, \kappa_{n}=\aleph_{n+1}$, and for all $1 \leq n<\omega$, there is no stationary subset of $\aleph_{n+1} \cap \operatorname{cof}\left(\aleph_{n}\right)$ which carries a partial square. For the first part, let $n<\omega$ be given. Then $\mathbb{Q}_{0} * \cdots * \mathbb{Q}_{n}$ forces $\kappa_{n}=\aleph_{n+1}$. It also forces that $\mathbb{P}_{n+1, \omega}$ is $\kappa_{n}$-closed. So $\mathbb{P}_{\omega}=\mathbb{Q}_{0} * \cdots * \mathbb{Q}_{n} * \mathbb{P}_{n+1, \omega}$ forces $\kappa_{n}=\aleph_{n+1}$.

For the second part, let $1 \leq n<\omega$ be given. Then $\mathbb{Q}_{0} * \cdots * \mathbb{Q}_{n}$ forces that there is no stationary subset of $\aleph_{n+1} \cap \operatorname{cof}\left(\aleph_{n}\right)$ which carries a partial square. It also forces $\mathbb{Q}_{n+1}$ that is $\kappa_{n+1}$-distributive, that is, $\aleph_{n+2}$-distributive, and hence adds no subsets of $\aleph_{n+1}$. Thus $\mathbb{Q}_{0} * \cdots * \mathbb{Q}_{n+1}$ forces no partial square. Since $\mathbb{P}_{n+2, \omega}$ is forced to be $\kappa_{n+1}$-closed, that is, $\aleph_{n+2}$-closed, $\mathbb{P}_{\omega}=\mathbb{Q}_{0} * \cdots * \mathbb{Q}_{n+1} * \mathbb{P}_{n+2, \omega}$ forces no partial square.

\section{Adding a Partial Square}

Let us now see that the naive approach for obtaining no partial squares on successive cardinals discussed in Section 2 does not work. Suppose that $\kappa<\kappa^{+}<\lambda$ are regular uncountable cardinals, where $\lambda$ is strongly inaccessible, and there is no partial square on any stationary subset of $\kappa^{+} \cap \operatorname{cof}(\kappa)$. We will show that the Lévy collapse $\operatorname{CoLL}\left(\kappa^{+},<\lambda\right)$ adds a stationary subset of $\kappa^{+} \cap \operatorname{cof}(\kappa)$ which carries a partial square.

The next proposition involves a variation of a forcing poset introduced in [2].

Proposition 5.1. Let $\kappa$ be a regular uncountable cardinal. Then there exists a $\kappa^{+}$-closed forcing poset which adds a stationary set $S \subseteq \kappa^{+} \cap \operatorname{cof}(\kappa)$ which carries a partial square.

Proof. Define $\mathbb{P}$ as the forcing poset whose conditions are sequences $\left\langle c_{\alpha}: \alpha \in A\right\rangle$ satisfying:

(1) $A \subseteq \kappa^{+} \cap \operatorname{cof}(\kappa)$;

(2) $|A| \leq \kappa ;$

(3) each $c_{\alpha}$ is a club subset of $\alpha$ with order type $\kappa$;

(4) if $\gamma \in \lim \left(c_{\alpha}\right) \cap \lim \left(c_{\beta}\right)$, then $c_{\alpha} \cap \gamma=c_{\beta} \cap \gamma$. 
Given conditions $p=\left\langle c_{\alpha}: \alpha \in A\right\rangle$ and $q=\left\langle d_{\beta}: \beta \in B\right\rangle$, let $q \leq p$ if $B$ is an end-extension of $A$, and for all $\alpha \in A, c_{\alpha}=d_{\alpha}$.

To see that $\mathbb{P}$ is $\kappa^{+}$-closed, let $\delta<\kappa^{+}$be a limit ordinal and let $\left\langle p_{i}: i<\delta\right\rangle$ be a descending sequence of conditions. By the definition of the ordering, we can write $p_{i}=\left\langle c_{\alpha}: \alpha \in A_{i}\right\rangle$ for each $i<\delta$. Let $A=\bigcup A_{i}$. Then $q=\left\langle c_{\alpha}: \alpha \in A\right\rangle$ is a condition in $\mathbb{P}$ and is a lower bound.

Let $\dot{S}$ be a $\mathbb{P}$-name such that $\mathbb{P}$ forces

$$
\dot{S}=\bigcup\{A: \exists p \in \dot{G} A=\operatorname{dom}(p)\} .
$$

Then $\mathbb{P}$ forces that $\dot{S}$ carries a partial square. It suffices to prove that $\dot{S}$ is forced to be stationary.

So assume that $p$ forces $\dot{C}$ is a club subset of $\kappa^{+}$, and we will find a condition $q \leq p$ and an ordinal $\alpha$ such that $q \Vdash \alpha \in \dot{S} \cap \dot{C}$. Fix a regular cardinal $\theta$ large enough so that $\mathbb{P}$ and $\dot{C}$ are in $H(\theta)$, and let $\unlhd$ be a well-ordering of $H(\theta)$.

Construct an internally approachable chain $\left\langle N_{i}: i\langle\kappa\rangle\right.$ of elementary substructures of the model $\langle H(\theta), \in, \unlhd, \mathbb{P}, p, \dot{C}\rangle$, each of size $\kappa$ and containing $\kappa$ as a subset. Let $N=\bigcup_{i} N_{i}$, and let $\alpha=N \cap \kappa^{+}$. Note that $\alpha$ has cofinality $\kappa$. Let $c=\left\{N_{i} \cap \kappa^{+}: i<\kappa\right\}$, so that $c$ is a club subset of $\alpha$ with order type $\kappa$.

Define by induction a descending sequence $\left\langle p_{i}: i<\kappa\right\rangle$ of conditions as follows. Let $p_{0}=p$. Suppose $p_{i}$ is defined, and assume by induction that $p_{i} \in N_{i+1}$. Since $\mathbb{P}$ is $\kappa^{+}$-closed, it is $\kappa^{+}$-distributive. As $N_{i}$ is in $N_{i+1}$ and has size $\kappa$, we can apply the $\kappa^{+}$-distributivity of $\mathbb{P}$ and let $p_{i+1}$ be the $\unlhd$-least condition below $p_{i}$ which is in every dense open subset of $\mathbb{P}$ in $N_{i}$. By elementarity, $p_{i+1}$ is in $N_{i+1}$.

Now suppose $\delta<\kappa$ is a limit ordinal and for all $i<\delta, p_{i}$ is defined and is in $N_{i+1}$. Moreover, assume that the sequence $\left\langle p_{i}: i<\delta\right\rangle$ is definable in $N$ from $\left\langle N_{i}: i<\delta\right\rangle$. Let $p_{\delta}^{\prime}=\bigcup_{i<\delta} p_{i}$; then the domain of $p_{\delta}^{\prime}$ is a subset of $N_{\delta} \cap \kappa^{+}$. Let $\gamma_{\delta}$ be the least ordinal of cofinality $\kappa$ greater than $N_{\delta} \cap \kappa^{+}$. Define $p_{\delta}$ as $\left\langle c_{\beta}: \beta \in \operatorname{dom}\left(p_{\delta}^{\prime}\right) \cup\left\{\gamma_{\delta}\right\}\right\rangle$, where $c_{\beta}=p_{\delta}^{\prime}(\beta)$ for $\beta \in \operatorname{dom}\left(p_{\delta}^{\prime}\right)$, and $c_{\gamma_{\delta}}$ is a club subset of $\gamma_{\delta}$ of order type $\kappa$ such that

$$
c_{\gamma_{\delta}} \cap\left(N_{\delta} \cap \kappa^{+}\right)=c \cap\left(N_{\delta} \cap \kappa^{+}\right)=\left\{N_{i} \cap \kappa^{+}: i<\delta\right\} .
$$

This is possible since this set has order type less than $\kappa$. By elementarity, $p_{\delta}$ is in $N_{\delta+1}$.

To see that $p_{\delta}$ is a condition, we only need to check the coherence requirement for $c_{\gamma_{\delta}}$. Suppose $c_{\gamma_{\delta}}$ and $c_{\xi}$ have some common limit point. By the minimality of $\gamma_{\delta}, \xi<N_{\delta} \cap \kappa^{+}$. So by the choice of $c_{\gamma_{\delta}}$, this common limit point is of the form $N_{\beta} \cap \kappa^{+}$for some limit ordinal $\beta<\delta$. As $\beta$ is a limit ordinal, $p_{\beta}$ was defined in the same manner as we described for $p_{\delta}$. Hence $N_{\beta} \cap \kappa^{+}$is a limit point of $c_{\gamma_{\beta}}$. But $\xi$ and $\gamma_{\beta}$ both appear in the domain of some condition. Therefore $c_{\xi} \cap\left(N_{\beta} \cap \kappa^{+}\right)=c_{\gamma_{\beta}} \cap\left(N_{\beta} \cap \kappa^{+}\right)=\left\{N_{i} \cap \kappa^{+}: i<\beta\right\}=c_{\gamma_{\delta}} \cap\left(N_{\beta} \cap \kappa^{+}\right)$.

This completes the definition of the sequence of conditions $\left\langle p_{i}: i<\kappa\right\rangle$. Let $A=\bigcup_{i<\kappa} \operatorname{dom}\left(p_{i}\right)$. Let $q=\left\langle c_{\zeta}: \zeta \in A \cup\{\alpha\}\right\rangle$, where $c_{\zeta}=p_{i}(\zeta)$ for some (any) $i<\kappa$ such that $\zeta \in \operatorname{dom}\left(p_{i}\right)$, and $c_{\alpha}=c$. The same argument as in the preceding paragraph shows that $q \in \mathbb{P}$. Clearly $q \leq p_{i}$ for all $i<\kappa$.

For each dense open set $D \in N$, there is $i<\kappa$ such that $p_{i} \in N \cap D$. It follows by standard arguments that $q$ forces that $\alpha$ is a limit point of $\dot{C}$, and hence is in $\dot{C}$. Also $q$ forces $\alpha \in \dot{S}$, because $\alpha$ is in the domain of $q$. Thus $q$ forces that $\alpha$ is in $\dot{S} \cap \dot{C}$. 
Let us go back to the situation described at the beginning of the section. Let $\kappa<\kappa^{+}<\lambda$ be regular uncountable cardinals, where $\lambda$ is strongly inaccessible. We will show that the Lévy collapse $\operatorname{CoLL}\left(\kappa^{+},<\lambda\right)$ introduces a stationary subset of $\kappa^{+} \cap \operatorname{cof}(\kappa)$ which carries a partial square.

Let $\mathbb{P}$ be the $\kappa^{+}$-closed forcing poset for adding a stationary subset of $\kappa^{+} \cap \operatorname{cof}(\kappa)$ which carries a partial square. Fix a regular cardinal $\theta<\lambda$ which is larger than $\kappa^{+}$ and the power set of $\mathbb{P}$. By Magidor's Theorem, we can factor $\operatorname{CoLL}\left(\kappa^{+}, \theta\right)$ as $\mathbb{P} * \mathbb{Q}$, where $\mathbb{Q}$ is forced to be $\kappa^{+}$-closed. It follows that the Lévy collapse $\operatorname{CoLL}\left(\kappa^{+},<\lambda\right)$ can be factored as

$$
\operatorname{CoLL}\left(\kappa^{+},<\lambda\right)=\mathbb{P} * \mathbb{Q} * \operatorname{CoLL}\left(\kappa^{+}, A\right),
$$

where $A=\lambda \backslash\{\theta\}$.

Let $G=H * I * J$ be a generic filter for $\operatorname{ColL}\left(\kappa^{+},<\lambda\right)=\mathbb{P} * \mathbb{Q} * \operatorname{CoLL}\left(\kappa^{+}, A\right)$. Then in $V[H]$, there is a stationary set $S \subseteq \kappa^{+} \cap \operatorname{cof}(\kappa)$ which carries a partial square. Since $S$ carries a partial square, $S$ is in the approachability ideal $I\left[\kappa^{+}\right]$, which implies that the stationarity of $S$ is preserved by $\kappa^{+}$-closed forcing (see [1]). As $\mathbb{Q} * \operatorname{CoLL}\left(\kappa^{+}, A\right)$ is $\kappa^{+}$-closed, $S$ remains stationary in $V[G]=V[H * I * J]$ and carries a partial square in that model.

Although the naive attempt to produce a model with successive cardinals satisfying no partial square does not work, we do not know what kind of large cardinals are necessary. For example, suppose for some regular uncountable cardinal $\kappa$, both $\kappa^{+} \cap \operatorname{cof}(\kappa)$ and $\kappa^{++} \cap \operatorname{cof}\left(\kappa^{+}\right)$have no stationary subsets which carry a partial square. Does it follow that $0^{\#}$ exists?

\section{REFERENCES}

[1] J. Cummings. Notes on singular cardinal combinatorics. Notre Dame J. Formal Logic, 46(3):251-282, 2005.

[2] J. Cummings and D. Wylie. More on full reflection below $\aleph_{\omega}$. Arch. Math. Logic, 49(6):659$671,2010$.

[3] J. Krueger and E. Schimmerling. An equiconsistency result on partial squares. J. Math. Log., 11(1):1-31, 2011.

[4] M. Magidor. Reflecting stationary sets. J. Symbolic Logic, 47(4):755-771, 1982.

[5] H. Sakai. Partial square at $\omega_{1}$ is implied by MM but not by PFA. Fund. Math., 215:109-131, 2011.

[6] S. Shelah. Reflecting stationary sets and successors of singular cardinals. Arch. Math. Logic, 31(1):25-53, 1991

[7] B. Veličković. Jensen's $\square$ principles and the Novák number of partially ordered sets. J. Symbolic Logic, 51(1):47-58, 1986

Department of Mathematics, University of North Texas

E-mail address: jkrueger@unt.edu 